\title{
What Borromini Might Have Known About Ovals. Ruler and Compass Constructions
}

\author{
Angelo Alessandro Mazzotti
}

Published online: 14 May 2014

(C) Kim Williams Books, Turin 2014

\begin{abstract}
This paper is about drawing ovals using a given number of certain parameters. New constructions are displayed, including the case when the symmetry axes are not given. Many of these constructions make use of a recent conjecture by Ragazzo, for which a Euclidean proof is found, thus suggesting it might have been known at the time Borromini chose the ovals for the dome of San Carlo alle Quattro Fontane. A geometric proof of the same conjecture-as well as constructions-in the more general case of eggs and polycentric curves is the subject of the first part of this same research (Mazzotti, a Euclidean approach to eggs and polycentric curves, 2014).
\end{abstract}

Keywords Borromini - San Carlo alle Quattro Fontane · Geogebra · Euclidean geometry $\cdot$ Ovals $\cdot$ Eggs $\cdot$ Polycentric curves

\section{Introduction}

At the beginning of the seventeenth century the young Francesco Castelli, born in Bissone (today Switzerland), moves to Milan to learn the skill of stonecutting, and then lands in Rome in the year 1619, eventually choosing for himself the name of Borromini, after having begun to work as an architect. His first independent job is the construction of the church of San Carlo alle Quattro Fontane (1638-1642)—also known as San Carlino-where he is challenged by the problem of building a dome (Fig. 1) with a given rectangle as frame for the impost, being on the other hand free

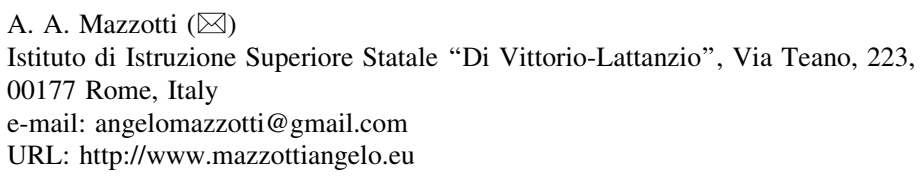




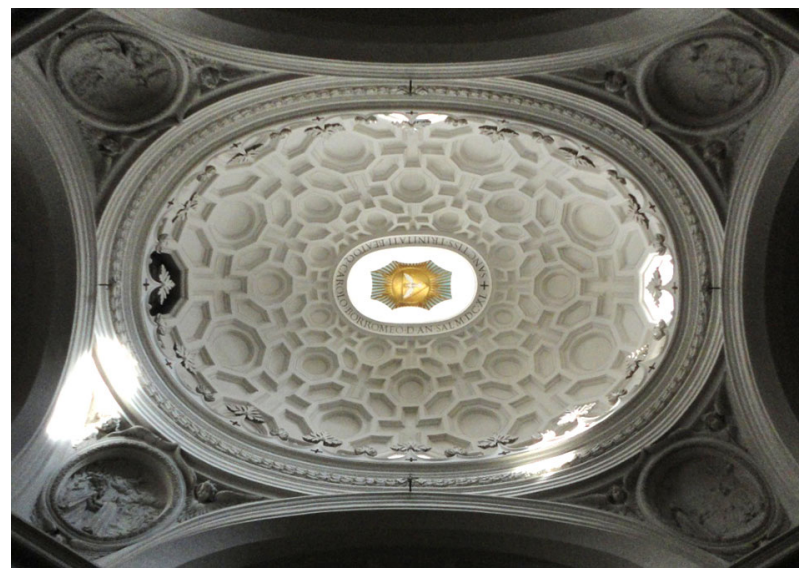

Fig. 1 The dome of S. Carlo alle Quattro Fontane, in Rome

to decide on how to close the dome, i.e. what kind of closed curves should be the narrowing horizontal sections.

The survey of the dome carried out by Alessandro Sartor, assisted by Margherita Caputo and Luisa Bogliolo in 1998, and the subsequent analysis I made with Margherita Caputo, confirmed that the chosen closed curves with two symmetry axes are all made of four smoothly connected arcs of circle, what is called a fourcentre oval, or simply an oval. This was no exception in the seventeenth century, although very recent studies found no evidence of constructions of ovals fitting any given proportion at the time San Carlino was built. Dotto (2001) reproduces Abraham Bosse's construction (Bosse 1655), among other ones, which could be the first published one actually dealing with ovals of any given proportion (see Fig. 2). The same method was displayed more than 50 years later by Tomás Vicente Tosca (see López Moto (2011) for references to both original text and electronic facsimile). At the time of Borromini, according to López Mozo, the only published constructions of ovals were those of Serlio, Ruiz, De l'Orme, and Vandelvira, covering only the cases of ratios between the major and the minor axis of $\sqrt{2}$, 1.3203, 1.3227, 1.4226, 2 and 1.5773 (in López Moto 2011; also Gentil Baldrich 1996). The proportion Borromini had to deal with to start building his dome was according to our calculations 1.4461 .

The fact that Borromini could not choose the constructions known at that time raised questions about what he gave himself as a rule to build the dome, how deep his knowledge about ovals was and how far he could have gone on his own. The analysis I made with M. Caputo on the San Carlino dome, as well as the work by Canciani and Spadafora (2012), shows that the artist was capable of drawing ovals that were not the ones that we today know had been published at the time. As can be clearly seen in Fig. 3, the shape of the impost is different from the fourth oval by Serlio (1619), which is the one published at the time that it most resembles: the centres of the arcs forming the top and bottom part of the oval of the impost lie on the vertical axis outside the curve, while the ones in Serlio's construction lie inside 


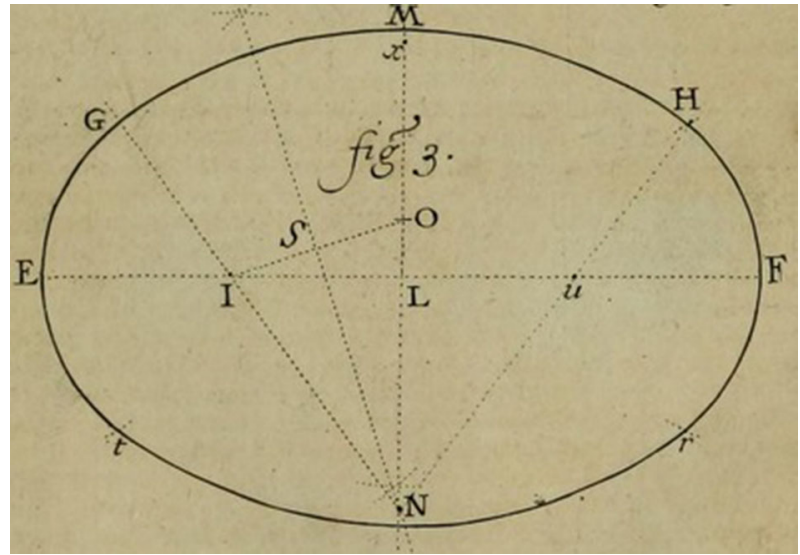

Fig. 2 Bosse's construction of an oval for any given axis measures and a choice for one of the centres (Bosse 1655)

the curve. Using Simona's words (2005), it is Borromini's "correction". The same remark on the originality of this shape can be found in the extensive work by Huerta (2007), which deals with both horizontal and vertical oval forms through history.

The problem of realising what artists and craftsmen knew about ovals at the time when they made use of them is still an open one. López Moto (2011), in her thorough and detailed work explains that the various "unconventional" ovals—such as those built in the Escorial 50 years before San Carlino-might not be necessarily only the effect of trial/error or approximation methods.

One of the aims of this paper is to show that ruler/compass constructions, given practically any choice of three parameters, are so straightforward that they could have been known, or easily found, already in ancient times - the mathematical tool for their proof being only Euclidean geometry-and that Borromini might have learned from sources unknown today, or discovered by himself, how to draw ovals enjoying properties he was interested in. He definitely took particular care in choosing shapes and ratios fitting both his aesthetic and his spiritual ideas, as explained by Hatch (2002) in his work on "Borromini's divine geometry". The core of this paper is a conjecture by Felice Ragazzo (1995) about the points where the arcs of circle of an oval meet, which I prove by means of Euclidean geometry, and which I use to generate a whole series of oval constructions. Both Bosse's construction-if he was interested in choosing one of the centres-or Ragazzo's construction-if he was interested in choosing the connection points of the arcs of circle-might have been at the time already proven and published by mathematicians, or fairly straightforward to be found by craftsmen. Architecture and mathematics historians are those entitled to go deeper in the subject. In the already mentioned works by Huerta (2007) and López Moto (2011) extensive bibliographical references on the subject of the use of ovals throughout history can be found.

The main theorem on ovals - the proof of the conjecture by Ragazzo (1995) — is derived from much more general theorems on eggs and polycentric curves, the subject of the paper of which this one is the continuation (Mazzotti 2014), inspired 

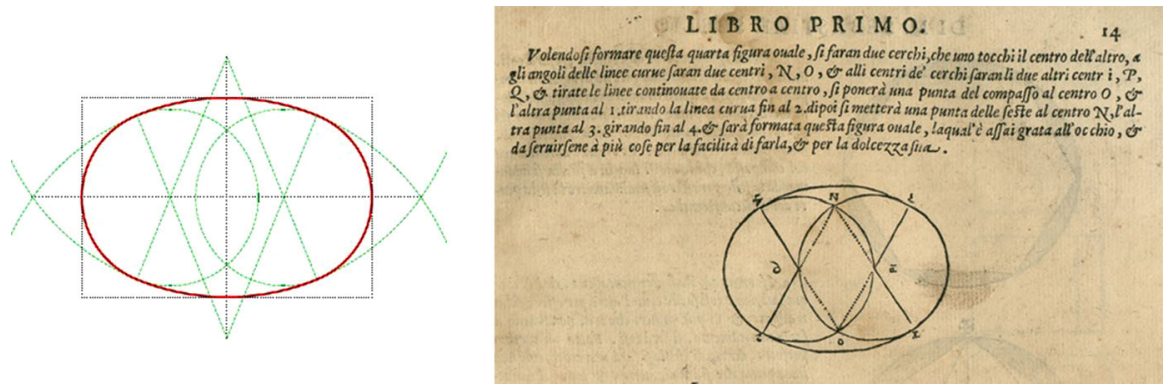

Fig. 3 The oval of the impost of the dome of San Carlino and the fourth construction by Sebastiano (1619). Image (left) by Margherita Caputo, used by permission

by Ragazzo's work on polycentric curves (2011). Only Euclidean geometry was used in order to be consistent with the above presented historical framework. The use of this conjecture gives way to a classification of oval constructions given any three parameters chosen in a proper way, both with and without indications on the directions of the axes, some of which are new. Freeware Geogebra has been extensively used for this purpose.

"The Euclidean Approach to the Study of Ovals" provides a quick reference to the properties of ovals. "The Main Theorems on Ovals. Properties of the Connection Locus" is devoted to the main theorem and to the properties of the set of points where the arcs of circle can meet - the Connection Locus. In "Constructing Ovals. Issues Still Open" I present a systematic list of different constructions of ovals, as well as issues still open. "Conclusions and Further Developments" is about my conclusions and my on-going research on the subject.

I have posted all of the constructions described on the internet. The complete list, the links and the descriptions are available on my website page http://www. mazzottiangelo.eu/en/pcc.asp.

\section{The Euclidean Approach to the Study of Ovals}

Being made of arcs of circle, and because of their symmetry, ovals can be easily studied by means of analytic geometry: Ragazzo's conjecture on ovals has already being proved by Ghione, in the Appendix of his paper (1995), using those tools. In the historical framework presented in the Introduction to this paper, I thought it more interesting to pretend Descartes and the other mathematicians responsible for the invention of the coordinate system had not been born yet.

Formulas derived via analytic geometry covering the whole topic of ovals will be the subject of my next paper.

For a clear and consistent approach to the subject I need to make clear what I mean here by oval:

an oval (or 4-centre oval or simple oval) is a non-twisted closed convex curve with two orthogonal symmetry axes (or simply axes) made of four arcs of circle subsequently smoothly connected, i.e. sharing a common tangent (Fig. 4). 


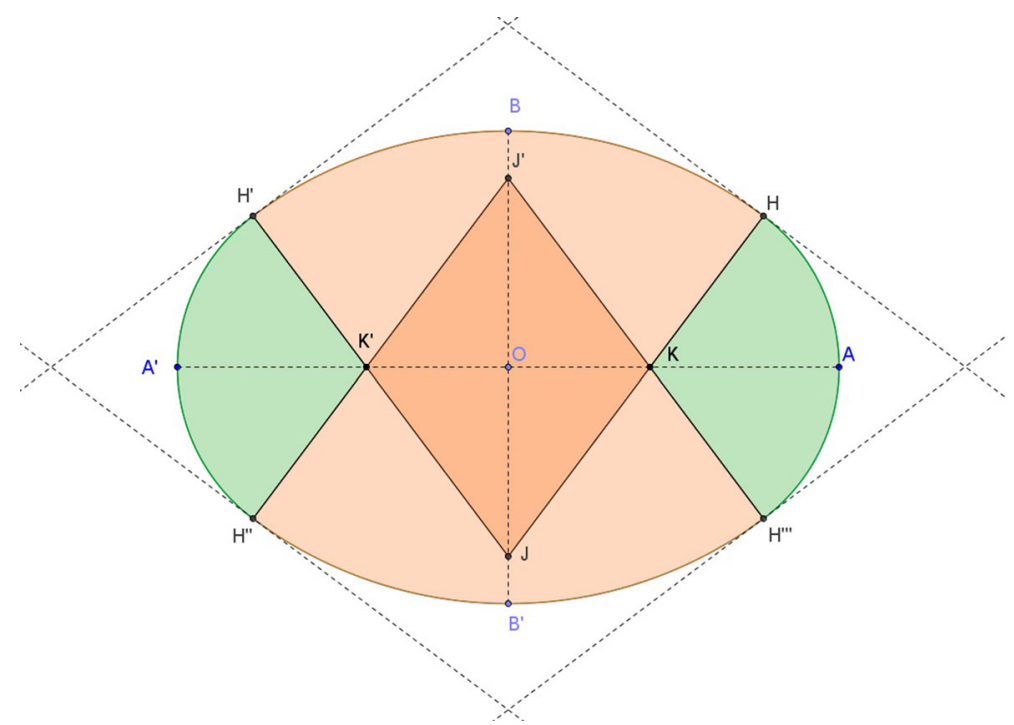

Fig. 4 An oval, with its four centres and the tangents at the connecting points

The double symmetry implies that, at least for the purposes of this paper, it is enough to deal with the top right-hand section, a quarter-oval. In this respect, using Fig. 5 as reference, I define

- $O$ as the intersection of the two symmetry axes

- $A$ and $B$ as the points of intersection between the quarter-oval and the horizontal and vertical axes. Let $\overline{O A}>\overline{O B}$

- $\quad K$ and $J$ as the centres, respectively, of the small and big circles, with radii $r_{1}$ and $r_{2}$, whose arcs form the quarter-oval

- $H$ as the connecting point of the two arcs

The above definitions and well-known Euclidean geometry theorems imply that:

- tangents to the circles in $A$ and $B$ are parallel to the other axis

- $J$ and $K$ belong, respectively, to the vertical and horizontal axis, with $K$ inside $O A$ and $J$ opposite to $B$ w.r.t. $O$

- $\quad J, K$ and $H$ are co-linear

Apart from the case $\overline{O A}=\overline{O B}$, where the oval becomes a circle, any other case allows for much more freedom than the ellipse-the similar but conceptually totally different well-known closed convex curve. An ellipse of given axes is totally determined by its rectangular frame, while there are infinitely many ovals having fixed points $A$ and $B$ (see Fig. 6): one has a further choice of either $J$ or $K$ or one of the distances of $H$ to the axes. In general one can fix (nearly) any three parameters and draw the corresponding oval. "Constructing Ovals. Issues Still Open" is devoted to these constructions and to the few unsolved cases. 
Fig. 5 A quarter oval
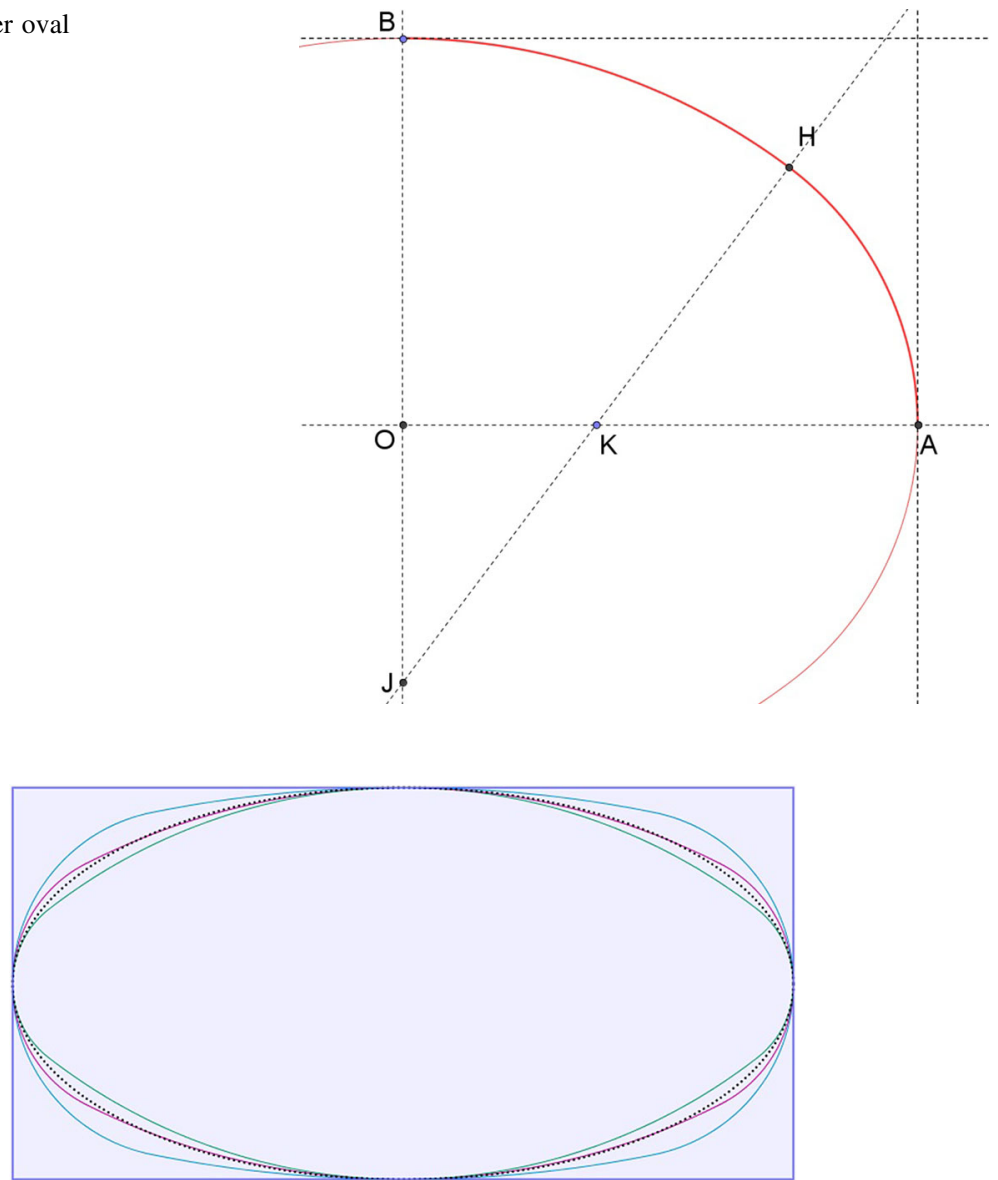

Fig. 6 In any given rectangle there is only one inscribed ellipse (the dotted curve), and an infinite number of ovals

Dotto's book on harmonic ovals (2002) deals with ovals with sizes with harmonic ratios.

\section{The Main Theorems on Ovals. Properties of the Connection Locus}

To be able to inscribe ovals in rectangles we want to be able to smoothly connect arcs of circles.

Ragazzo's conjecture on ovals reduces the problem to quarter ovals inside rectangles. It is the following (my translation from the Italian):

The locus of the connection points for the arcs (of a quarter oval [Ed.]) [...] is the circle, which I will call Connection Locus, defined through the following three points [points 1, 2 and 3 on Ragazzo's original drawing (Fig. 7)] 

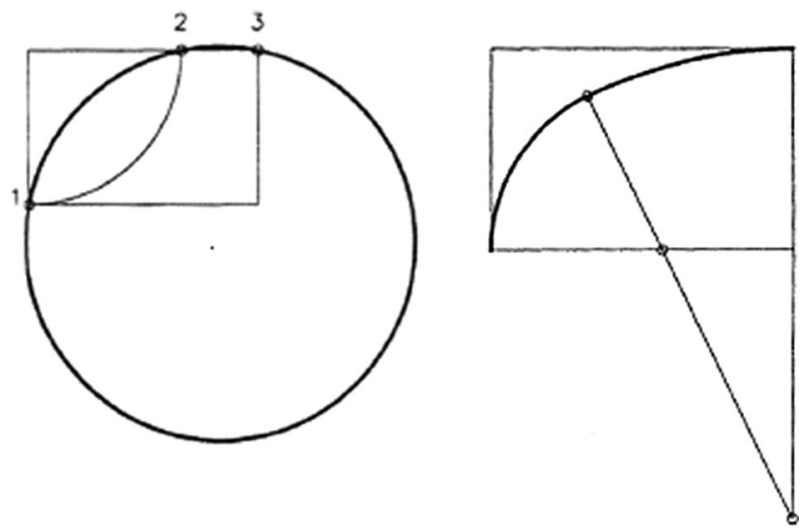

Fig. 7 Inscribing a quarter oval inside a rectangle: Ragazzo's circle through points 1, 2 and 3 and the quarter oval resulting from the choice of a connection point on it. Image (Ragazzo 1995), reproduced by permission

- intersection between the half major axis and the half minor side (of the rectangle inscribing the oval [Ed.])

- endpoint of the half minor side transported compass-wise onto the major side

- intersection between the half major side and the half minor axis

(Taken from Ragazzo 1995)

I first wrote the proof of this conjecture, then managed to extend it first to eggs and then to polycentric curves. So it has become a corollary of one of the theorems contained in my preceding paper (Mazzotti 2014).

Theorem 1 Let OATB be a rectangle with $\overline{B T}>\overline{A T}$ (see Fig. 8). Let $S$ be the point on BT such that $\overline{S T}=\overline{A T}$. Necessary and sufficient condition for $H$ to be the connecting point for two arcs of circle with centres inside B $\hat{T} A$ and tangent in $H$, one tangent to the line AT in A and the other tangent to the line TB in B, is for H to belong to the open arc AS of the circle through $A, B$ and $S$. Moreover, the angle originating at the centre of such a circle corresponding to the arc $A B$ is a right angle.

Proof Adirect application of Theorem 2 in Mazzotti (2014).

Definition I will call the circle through $A, B$ and $S$, following Ragazzo's work (1995), from now on Connection Locus (my translation), or simply CL.

The remark just after Theorem 2 in Mazzotti (2014) implies very important properties of point $R$ :

- the line $R T$ forms a $\frac{\pi}{4}$ angle with $A O$ and passes through $V$

- $R M, M$ being the midpoint of $O V$, is the axis of $O V$, therefore triangle $O R V$ is isosceles and $R \hat{O} V$ is also equal to $\frac{\pi}{4}$

- $R K$ bisects $A \hat{K} H$

- $R J$ bisects $H \hat{J} B$

Summing up these properties of point $R$ for quarter ovals we have the following. 
Fig. 8 The quarter oval resulting from a choice of $H$ on the Connection Locus of the given rectangle

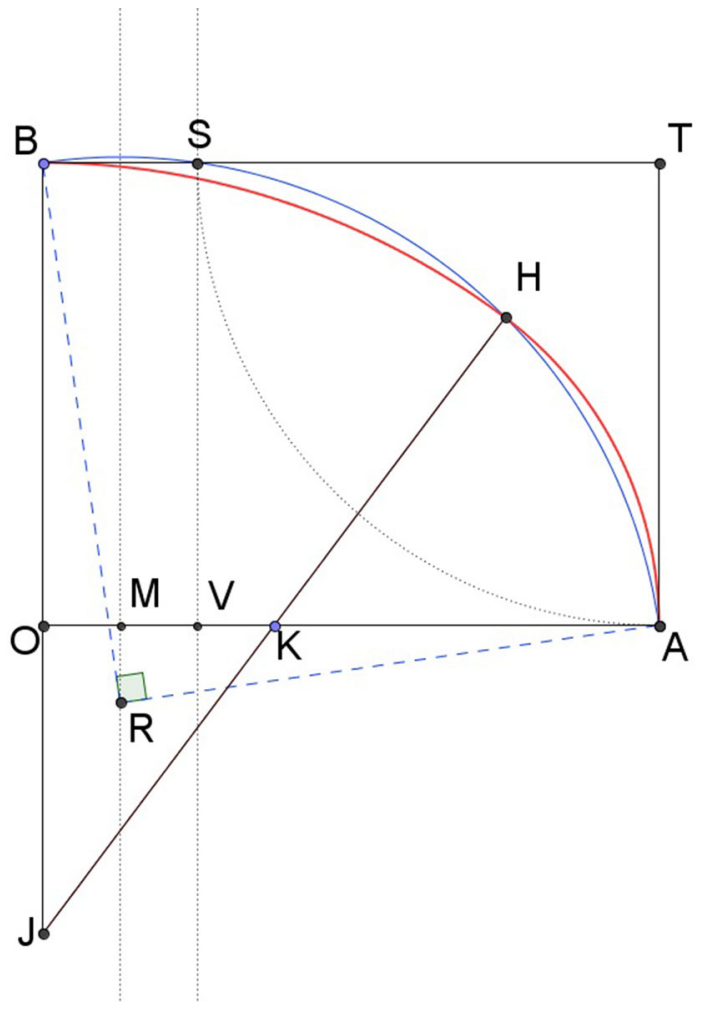

Theorem 2 The centre $R$ of the Connection Locus for a quarter oval is the incentre of triangle OKJ.

The fact that $R O$ bisects $J \hat{O} A$ is another result already conjectured by Ragazzo (1995).

\section{Constructing Ovals. Issues Still Open}

We are now ready to show how nearly any oval can be drawn if enough parameters are known. All the constructions I will talk about have been made with freeware Geogebra and are linked through my website http://www. mazzottiangelo.eu/en/pcc.asp, as described further. The heavily used Connection Locus - the $C L$ - has just been defined in "The Main Theorems on Ovals. Properties of the Connection Locus".

Issues left open are the "missing" ruler/compass constructions of Ovals 8, 10, 11 and 13, as well as some kind of proof of the suggested limitations for the parameters in the constructions listed in "Ovals Without Given Symmetry Axis Lines". 


\section{Ovals With Given Symmetry Axis Lines}

First of all we will deal with ovals with known symmetry axis lines. Let $O$ be the symmetry centre. Constructions 21 and 22 are to my knowledge totally new and justified by mathematical arguments.

Considering only a quarter oval we will use the following parameter names (see Fig. 5). Let

- $a=\overline{O A}$ the length of half the major axis

- $b=\overline{O B}$ the length of half the minor axis

- $k=\overline{O K}$ the distance from $O$ of the centre of the smaller circle

- $j=\overline{O J}$ the distance from $O$ of the centre of the bigger circle

- $h$ the distance of the connecting point $H$ to $O B$

- $m$ the distance of the connecting point $H$ to $O A$

- $\quad r_{1}$ the measure of the radius $\overline{A K}$

- $r_{2}$ the measure of the radius $\overline{B J}$

- $\beta=A \hat{K} H$ the angle formed by the line of the centres and the major axis

- $p=\frac{\overline{O B}}{\overline{O A}}$ the ratio of the two axes

My conjecture is that:

The first six parameters are independent of one another, and any choice of three of them-constrained within certain values—will determine a unique oval.

I will show this producing 16 out of 20 compass/ruler constructions and showing that the four remaining, although yet to be found, correspond to uniquely determined ovals. I will after this show other constructions-some of them newinvolving parameters $\beta, p, r_{1}$ and $r_{2}$.

Figure 9 lists the 20 different choices of three out of six of the above parameters, and the number of the corresponding oval, for further easier references.

In the following constructions some parameters are subject to constraints, but there exist always feasible values. Precise limitations - via analytic calculationswill be listed in my next paper on the subject.

Construction of Oval 1 (Fig. 10)—given $a, b$ and $k$-uses Ragazzo's CL and is thus mathematically supported also by Theorem 1 (see also link to Geogebra animation video on my website http://www.mazzottiangelo.eu/en/pcc.asp); other possibilities include Bosse's method (1655) (see also for example Dotto 2001) or another method inspired by Archimede's lemma (see Ragazzo 1995);

- draw the perpendiculars to $O A$ through $A$, and to $O B$ through $B$; let $T$ be their intersection

- $\quad$ find $S$ inside segment $T B$ such that $\overline{T S}=\overline{T A}$

- draw the circle through $A, B$ and $S$-the CL

- draw the circle with radius $\overline{A K}$ and centre $K$ and let $H$ be the intersection between the two

- let $J$ be the intersection of lines $K H$ and $O B$

- $\quad \operatorname{arc} H B$ with centre $J$ and arc $A H$ with centre $K$ form the quarter-oval 


\begin{tabular}{|c|c|c|c|c|c|c|c|c|c|c|c|c|c|c|c|c|c|c|c|c|}
\hline oral & 1 & 2 & 3 & 4 & 5 & 6 & 7 & 8 & 9 & 10 & 11 & 12 & 13 & 14 & 15 & 16 & 17 & 18 & 19 & 20 \\
\hline $\mathrm{a}$ & $\bullet$ & $\bullet$ & $\bullet$ & $\bullet$ & $\bullet$ & $\bullet$ & $\bullet$ & $\bullet$ & $\bullet$ & $\bullet$ & & & & & & & & & & \\
\hline $\mathrm{b}$ & $\bullet$ & $\bullet$ & $\bullet$ & $\bullet$ & & & & & & & $\bullet$ & $\bullet$ & $\bullet$ & $\bullet$ & $\bullet$ & $\bullet$ & & & & \\
\hline $\mathrm{k}$ & $\bullet$ & & & & $\bullet$ & $\bullet$ & $\bullet$ & & & & $\bullet$ & $\bullet$ & $\bullet$ & & & & $\bullet$ & $\bullet$ & $\bullet$ & \\
\hline $\mathrm{h}$ & & $\bullet$ & & & $\bullet$ & & & $\bullet$ & $\bullet$ & & $\bullet$ & & & $\bullet$ & $\bullet$ & & $\bullet$ & $\bullet$ & & $\bullet$ \\
\hline $\mathrm{j}$ & & & $\bullet$ & & & $\bullet$ & & $\bullet$ & & $\bullet$ & & $\bullet$ & & $\bullet$ & & $\bullet$ & $\bullet$ & & $\bullet$ & $\bullet$ \\
\hline $\mathrm{m}$ & & & & $\bullet$ & & & $\bullet$ & & $\bullet$ & $\bullet$ & & & $\bullet$ & & $\bullet$ & $\bullet$ & & $\bullet$ & $\bullet$ & $\bullet$ \\
\hline
\end{tabular}

Fig. 9 The different oval construction nos. according to the given parameters, when the symmetry axes lines are known

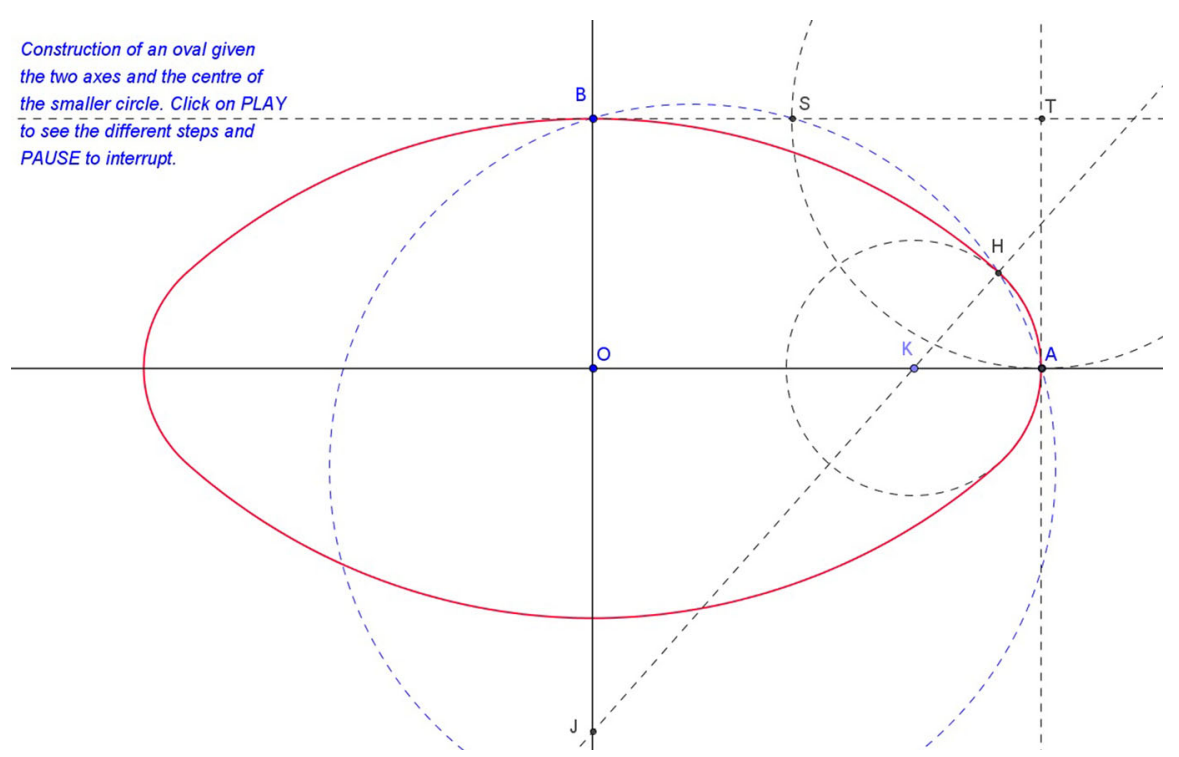

Fig. 10 Constructing Oval 1

Construction of Oval 2 (Fig. 11) — given $a, b$ and $h$-also uses the CL (see link to Geogebra animation video on my website http://www.mazzottiangelo.eu/en/pcc. asp) :

- draw the perpendiculars to $O A$ through $A$, and to $O B$ through $B$; let $T$ be their intersection

- $\quad$ find $S$ inside segment $T B$ such that $\overline{T S}=\overline{T A}$

- draw the circle through $A, B$ and $S$-the CL

- find the only point $H$ on arc $A S$ to have distance $h$ from $O A$

- draw the axes of segment $A H$ and let $K$ be the intersection with $O A$

- let $J$ be the intersection of lines $K H$ and $O B$

- $\quad \operatorname{arc} H B$ with centre $J$ and $\operatorname{arc} A H$ with centre $K$ form the quarter-oval 


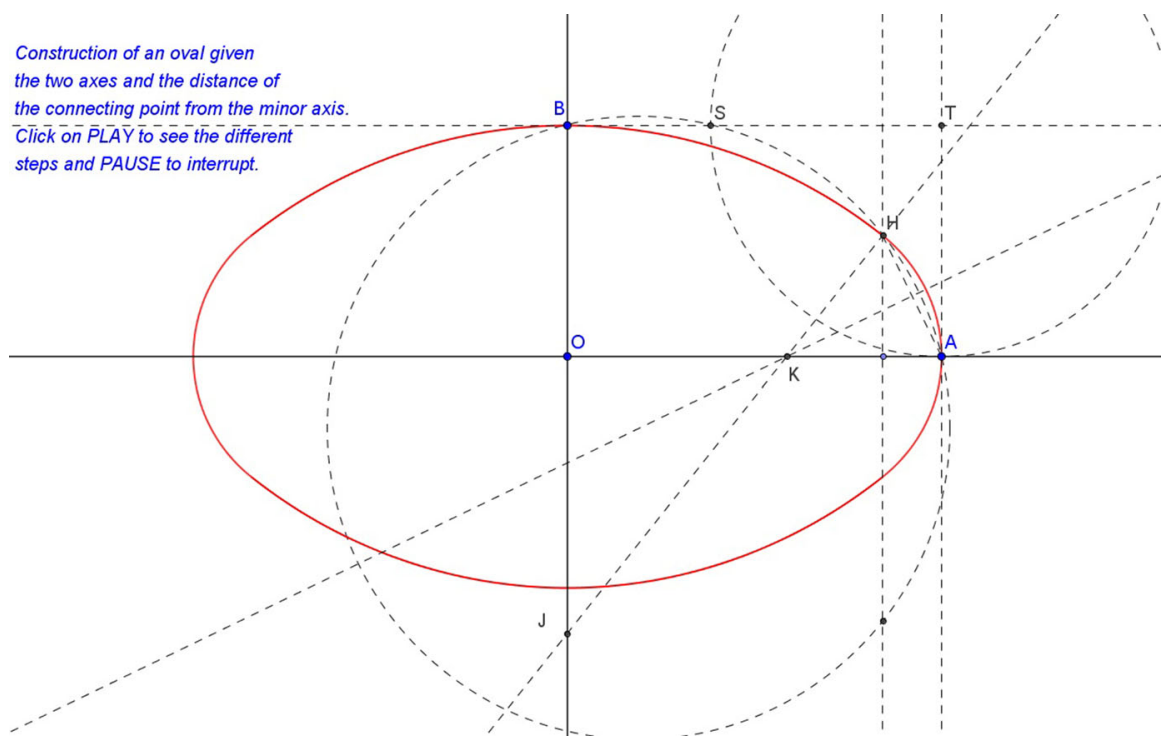

Fig. 11 Constructing Oval 2

Construction of Oval 3-given $a, b$ and $j$-proceeds like Oval 1 (see link to Geogebra animation video on my website http://www.mazzottiangelo.eu/en/pcc. asp).

Construction of Oval 4-given $a, b$ and $m$-proceeds like Oval 2 (see link to Geogebra animation video on my website http://www.mazzottiangelo.eu/en/pcc. asp).

Construction of Oval 5 (Fig. 12) — given $a, k$ and $h$-is straightforward (see link to Geogebra animation video on my website http://www.mazzottiangelo.eu/en/pcc. asp):

- $H$ is the point inside the right angle $A \hat{O} B$ on the circle with radius $\overline{A K}$ and centre $K$ having distance $h$ from $O B$

- let $J$ be the intersection of lines $K H$ and $O B$

- an arc with centre $J$ and radius $J H$ till the intersection $B$ with the vertical axis, and $\operatorname{arc} A H$ with centre $K$ form the quarter-oval

Construction of Oval 6 (Fig. 13) - given $a, k$ and $j$-is also straightforward (see link to Geogebra animation video on my website http://www.mazzottiangelo.eu/en/ pcc.asp):

- $H$ is the intersection inside the right angle $A \hat{O} B$ of $J K$ and the circle with radius $\overline{A K}$ and centre $K$

- an arc with centre $J$ and radius $J H$ till the intersection $B$ with the vertical axis, and $\operatorname{arc} A H$ with centre $K$ form the quarter-oval 


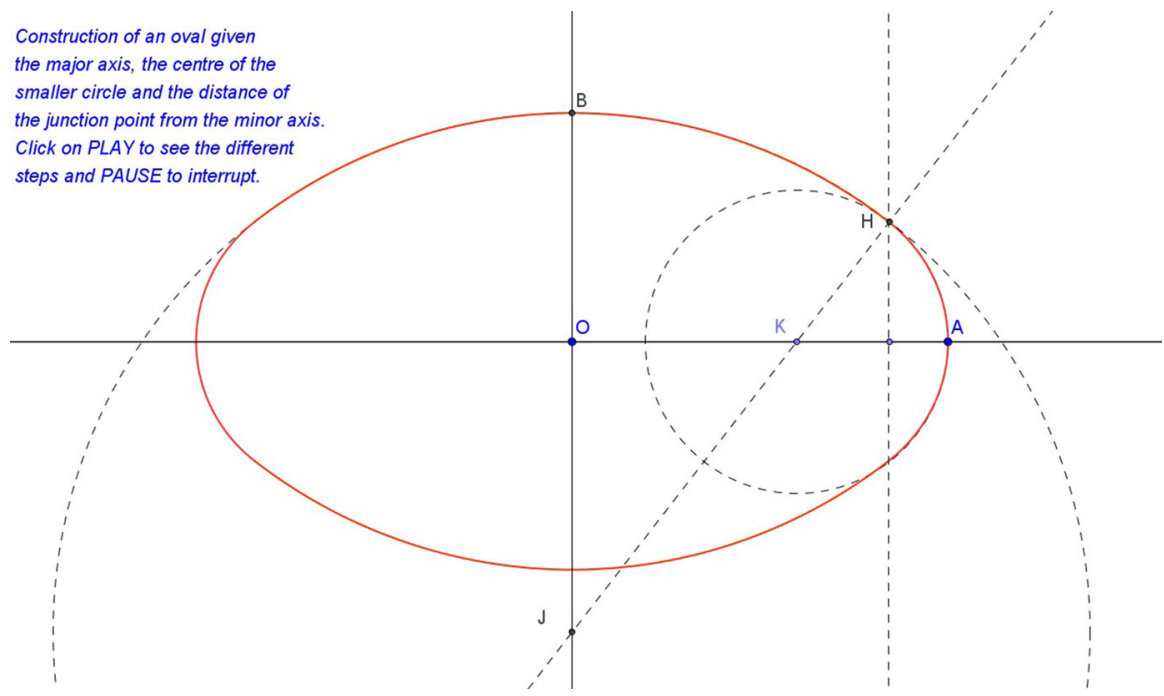

Fig. 12 Constructing Oval 5

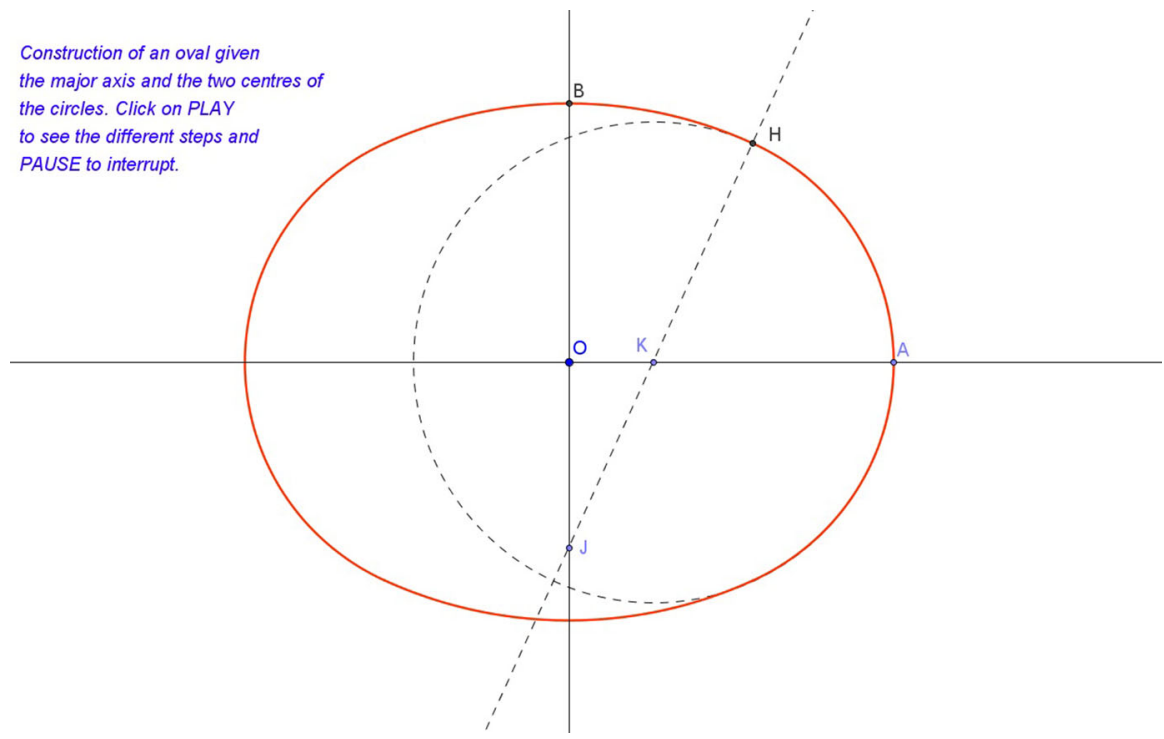

Fig. 13 Constructing Oval 6

Construction of Oval 7 (Fig. 14)_-given $a, k$ and $m$-is as follows (see link to Geogebra animation video on my website http://www.mazzottiangelo.eu/en/pcc. asp):

- $H$ is the nearest point to $A$ on the circle with radius $\overline{A K}$ and centre $K$ having distance $m$ to $O A$ 


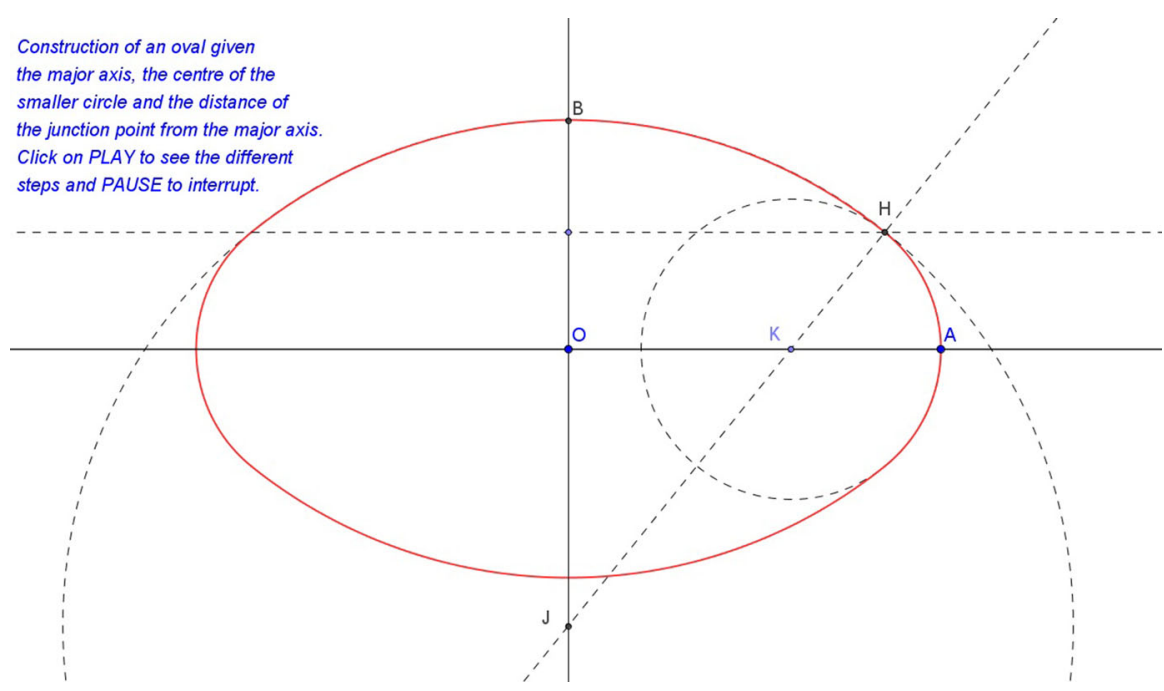

Fig. 14 Constructing Oval 7

- let $J$ be the intersection of lines $K H$ and $O B$

- an arc with centre $J$ and radius $J H$ till the intersection $B$ with the vertical axis, and $\operatorname{arc} A H$ with centre $K$ form the quarter-oval

Construction of Oval 8 -given $a, h$ and $j$-using compass and ruler remains at this moment unsolved, although there is graphic and algebraic evidence (analytic formulas will be displayed in my next paper) of the unicity of the corresponding oval. It is together with ovals 10,11 and 13-also unsolved-a "mixed data" case, where one of the known parameters is the centre of a circle, the second is the half axis on the other orthogonal line and the third is the distance of the connecting point to either of the axes. Solution to any of these cases would probably lead to the solution of the other three.

Construction of Oval 9 (Fig. 15)_-given $a, h$ and $m$-is as follows (see link to Geogebra animation video on my website http://www.mazzottiangelo.eu/en/pcc. asp):

- $K$ is the intersection between the axis of $A H$ and $O A$

- let $J$ be the intersection of lines $K H$ and $O B$

- an arc with centre $J$ and radius $J H$ till the intersection $B$ with the vertical axis, and $\operatorname{arc} A H$ with centre $K$ form the quarter-oval

Construction of Oval 10-given $a, j$ and $m$-using compass and ruler remains at this moment unsolved (see further remarks for the construction of Oval 8).

Construction of Oval 11 -given $b, k$ and $h$-using compass and ruler remains at this moment unsolved (see further remarks for the construction of Oval 8).

Construction of Oval 12-given $b, k$ and $j$-proceeds like Oval 6 (see link to Geogebra animation video on my website http://www.mazzottiangelo.eu/en/pcc. asp). 


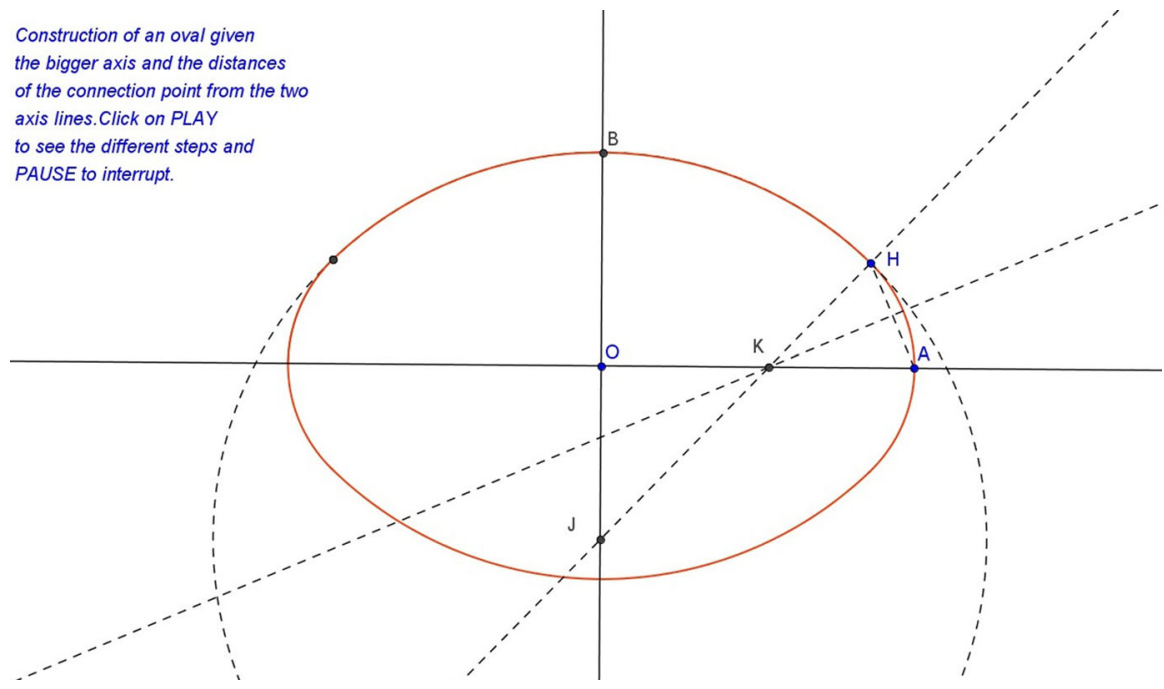

Fig. 15 Constructing Oval 9

Construction of Oval 13-given $b, k$ and $m$-using compass and ruler remains at this moment unsolved (see further remarks for the construction of Oval 8).

Construction of Oval 14-given $b, h$ and $j$-proceeds like Oval 5 (see link to Geogebra animation video on my website http://www.mazzottiangelo.eu/en/pcc. asp).

Construction of Oval 15-given $b, h$ and $m$-proceeds like Oval 9 (see link to Geogebra animation video on my website http://www.mazzottiangelo.eu/en/pcc. asp).

Construction of Oval 16-given $b, j$ and $m$-proceeds like Oval 7 (see link to Geogebra animation video on my website http://www.mazzottiangelo.eu/en/pcc. asp).

Construction of Oval 17 (Fig. 16) - given $k, h$ and $j$-is as follows (see link to Geogebra animation video on my website http://www.mazzottiangelo.eu/en/pcc. asp):

- $H$ is the point on the line $J K$ with distance $h$ from $O B$

- an arc with centre $J$ and radius $J H$ till the intersection $B$ with the vertical axis, and an arc with centre $K$ and radius $K H$ till the intersection $A$ with the horizontal axis form the quarter-oval

Construction of Oval 18 (Fig. 17) — given $k, h$ and $m$-is as follows (see link to Geogebra animation video on my website http://www.mazzottiangelo.eu/en/pcc. asp):

- let $J$ be the intersection of lines $K H$ and $O B$

- an arc with centre $J$ and radius $J H$ till the intersection $B$ with the vertical axis, and an arc with centre $K$ and radius $K H$ till the intersection $A$ with the horizontal axis form the quarter-oval 


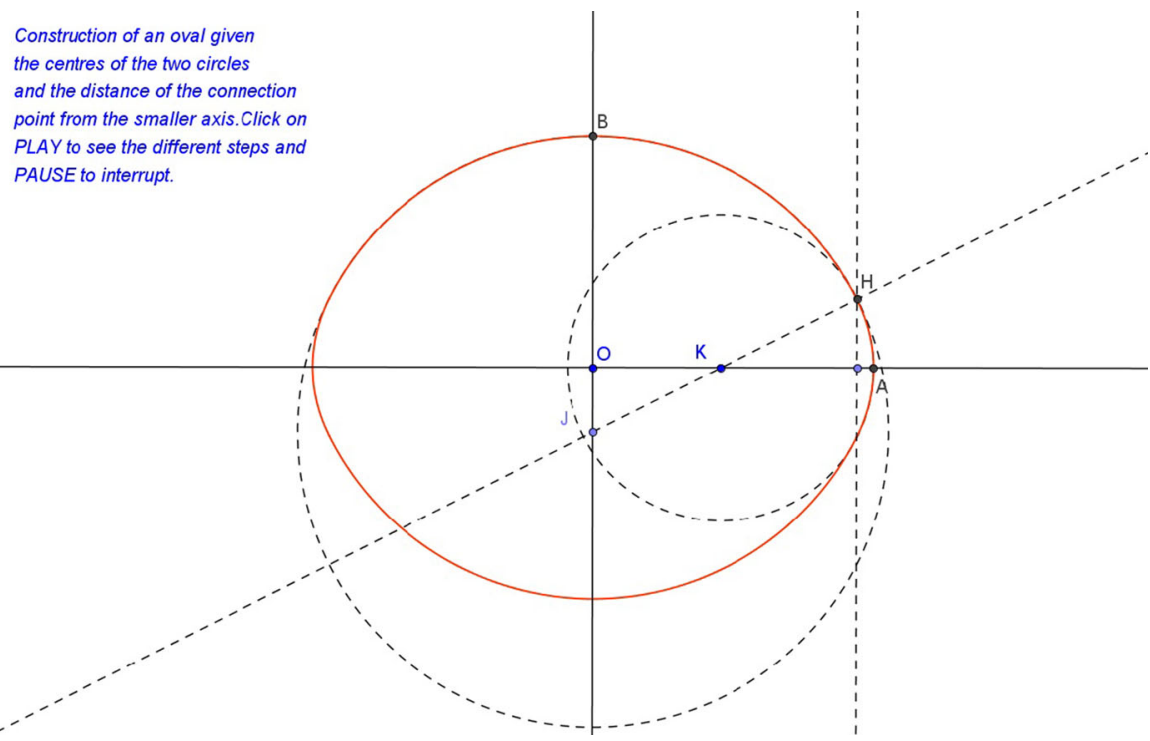

Fig. 16 Constructing Oval 17

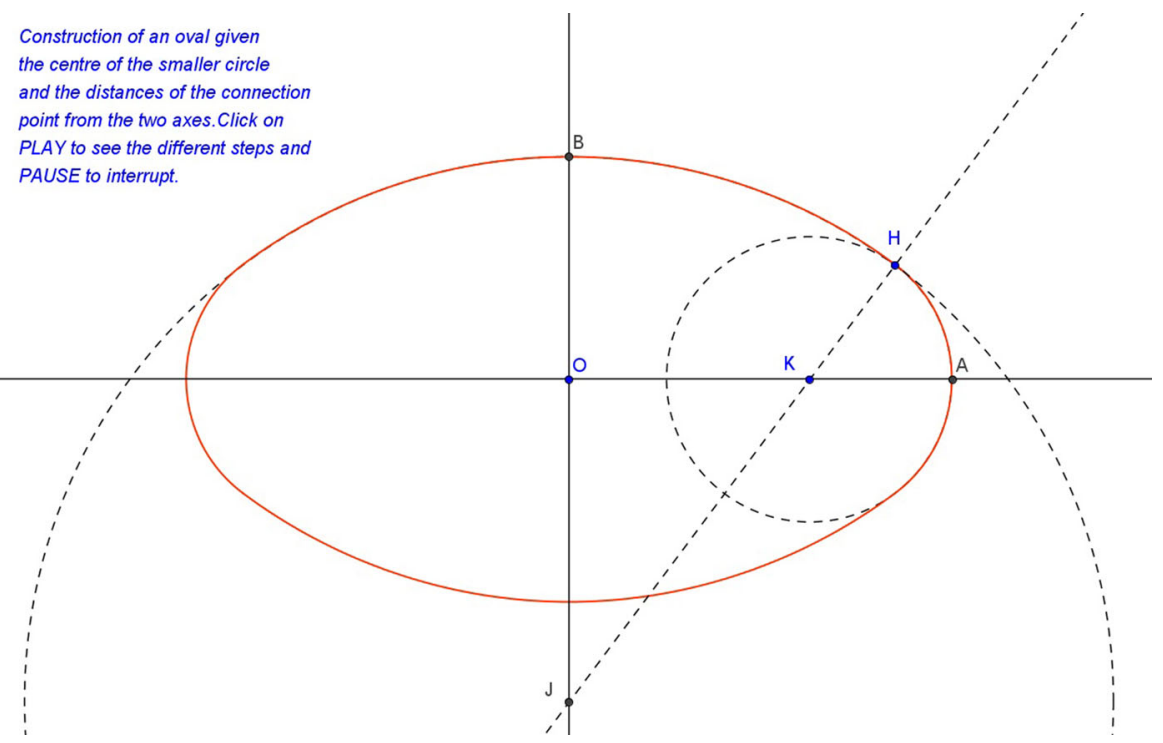

Fig. 17 Constructing Oval 18

Construction of Oval 19-given $k, j$ and $m$-proceeds like Oval 17 (see link to Geogebra animation video on my website http://www.mazzottiangelo.eu/en/pcc. asp). 
Construction of Oval 20-given $h, j$ and $m$-proceeds like Oval 18 (see link to Geogebra animation video on my website http://www.mazzottiangelo.eu/en/pcc. asp).

All the above constructions are justified by Theorem 1 and by the properties of ovals listed in "The Euclidean Approach to the Study of Ovals".

The following three use a different choice of parameters.

Oval 21 is constructed using any choice of (half) axis measures and any choice of the angle formed by the lines of the centres with the horizontal axis. It is similar to the generalised version of Christiaan Huygens' (1629-1695) construction (see for example Dotto 2001, 2002) although I use the CL. Figure 18 shows us that connection point $H$ is the (only) point on the CL, base vertex of an isosceles triangle with vertex $J$ and third vertex $B$, similar to the triangle $O A D$ having vertex $O$ and vertex angle complementary to the desired angle $\beta$. The $\mathrm{CL}$ is drawn considering that its centre $R$ is the vertex of an isosceles right triangle with other vertices $A$ and $B$ (see Theorem 1).

Construction of Oval 21 (Fig. 18) — given $a, b$ and $\beta$-is as follows (see link to Geogebra animation video on my website http://www.mazzottiangelo.eu/en/pcc. asp):

- draw a half line with origin $O$ and the given angle $\beta$

- with centre $O$ draw an arc with radius $b$ intersecting the halfline in $D$

- let $R$ be the vertex of the isosceles right triangle with hypotenuse $A B$ (the one on the same side of $O$ w.r.t. $A B$

- draw the CL with centre $R$ and radius $R B$, let $H$ be the intersection with $B D$

- draw from $H$ the parallel to $O D$; intersections with the axes are the centres $K$ and $J$ of the arcs forming the quarter-oval

In order for $B D$ to meet the CL between $A$ and $S$ (see once more Theorem 1 and Fig. 8) it must be $O \hat{B} A<O \hat{B} D<\frac{\pi}{2}$, which yields

$$
\operatorname{arctg} \frac{a}{b}<\frac{\pi-D \hat{O} B}{2}=\frac{\pi-\left(\frac{\pi}{2}-\beta\right)}{2}=\frac{\pi}{4}+\frac{\beta}{2}<\frac{\pi}{2}
$$

giving the following limitation for $\beta$ :

$$
2 \operatorname{arctg} \frac{a}{b}-\frac{\pi}{2}<\beta<\frac{\pi}{2}
$$

Construction 22 is to my knowledge also a new construction, building the quarter-oval given the centres of the arcs and the ratio $p$ between the half axes. Theorem 2 implies that point $R$ is the intersection between the bisectors of $O \hat{K} J$ and $K \hat{O} J$; that $T$ is on the line through $R$ forming an angle of $\frac{\pi}{4}$ with $O K$ has been shown in the remarks preceding Theorem 2.

Construction of Oval 22 (Fig. 19)-given $k, j$ and $p$-is as follows (see link to Geogebra animation video on my website http://www.mazzottiangelo.eu/en/pcc. asp):

- having fixed $K, J$ and the halfline with origin $O$ along which point $T$ is to be found, start by finding the incentre $R$ of triangle $O K J$ 


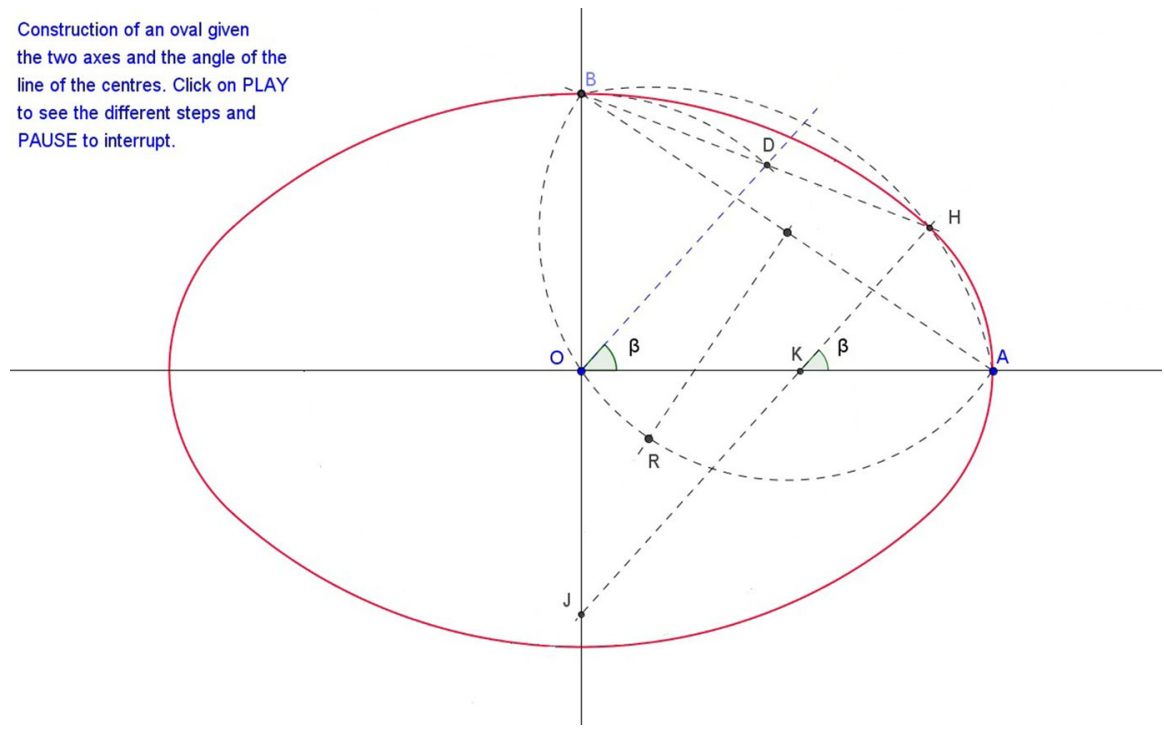

Fig. 18 Constructing Oval 21

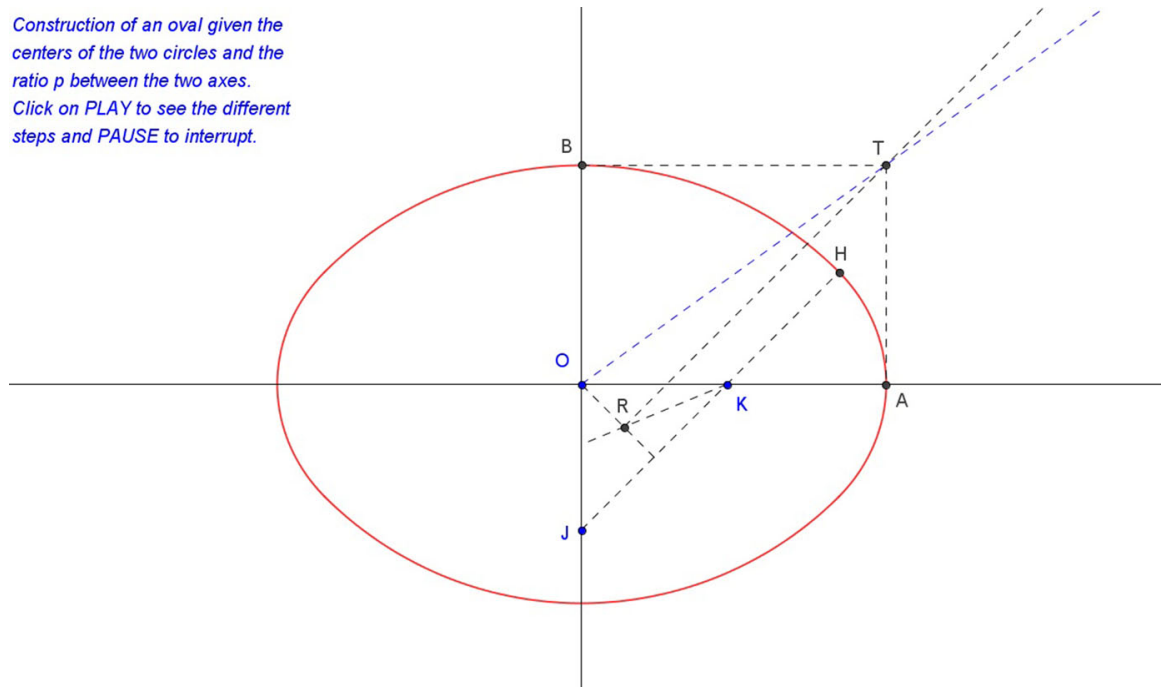

Fig. 19 Constructing Oval 22

- the intersection between the orthogonal line to $O R$ from $R$ and the half line with origin $O$ yield point $T$

- points $A$ and $B$ and the arcs forming the quarter-oval can now be drawn

Limitations for the value $p$ have been found via analytic calculations, they are therefore the subject of my next paper. 
Drawing an oval using the radii and one of the centres is fairly simple.

Construction of Oval 23 (Fig. 20)-given $r 1, r 2$ and $k$-is as follows (see link to Geogebra animation video on my website http://www.mazzottiangelo.eu/en/pcc. asp):

- having fixed $K$, draw a circle with radius $r_{2}-r_{1}$ and find the intersection $J$ with the vertical axis

- the two arcs can now be drawn

\section{Ovals Without Given Symmetry Axis Lines}

I will now consider the problem of drawing an oval on a plane without any given symmetry axes. We now have three more degrees of freedom and so a much bigger variety of combinations. I will nevertheless show with a few examples that parameters that look independent aren't always so.

The CL and the constructions used in the first part of this section are the tools to tackle this problem. Limitations conjectured for the parameters involved are maybe the most interesting part. I consider constructions 120,121 and 122 to be the most innovative ones.

Figure 21 is going to be my new reference.

To my experience an oval in a plane is determined by six independent parameters, although limitations do occur. I will again try to be systematic and give numbers to the different problems/solutions.

In what follows $A$ and $A^{\prime}$ are opposite vertices on the longer axis, $B$ and $B^{\prime}$ on the shorter, $J$ and $J^{\prime}$ centres of the arcs with bigger radius, $K$ and $K^{\prime}$ of the ones with smaller radius, and finally $H, H^{\prime}, H^{\prime \prime}$ and $H^{\prime \prime \prime}$ the connection points. I will again use $R$ to indicate the centre of a CL.

Given on a plane $A$ and $A^{\prime}$ opposite vertices - corresponding to four parametersthe symmetry centre $O$ will be their midpoint, and lines $A A^{\prime}$ and its orthogonal through $O$ will be the symmetry axes. One continues now by choosing any two parameters other than $a$ from Fig. 9 using the corresponding constructions already listed (excluding the unsolved cases). The ten possible choices will be numbered from 100 to 109 following the order in which they appear in Fig. 9.

Construction of Ovals 100 to 109 -given $A, A^{\prime}$ and any other two independent parameters other than $a$-follows the above guidelines once $O$ and the two axes have been drawn. As an example I will draw Oval 106 (Fig. 22), given $A, A^{\prime}, k$ and $m$ (see link to Geogebra animation video on my website http://www.mazzottiangelo. eu/en/pcc.asp):

- $O$ is the midpoint of $A A^{\prime}$, from which we draw the orthogonal to it

- a parallel distant $m$ from $A A^{\prime}$, and the circle with centre a point $K$-distant $k$ from $O$ on $A A^{\prime}$-meet in $H$.

- we find now $J$ intersecting $H K$ and the drawn orthogonal to $A A^{\prime}$, and draw the complete oval as usual, using symmetry properties

The same ideas allow for constructions when $B$ and $B^{\prime}$ are given. 


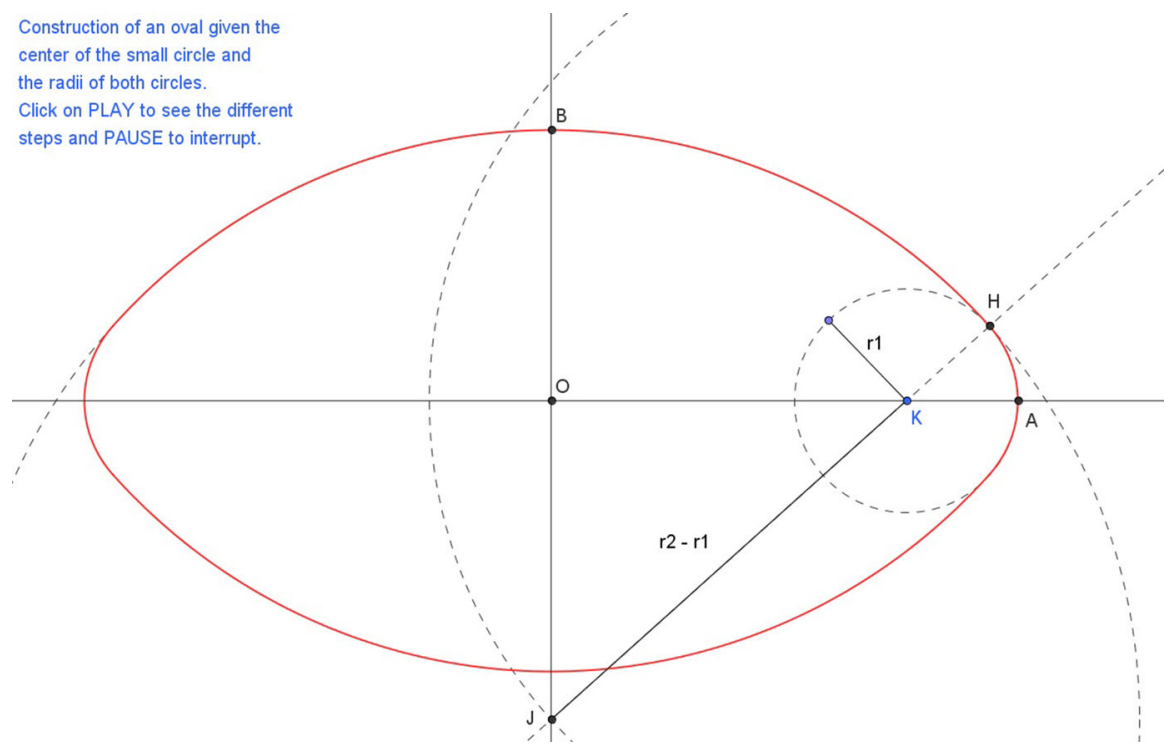

Fig. 20 Constructing Oval 23

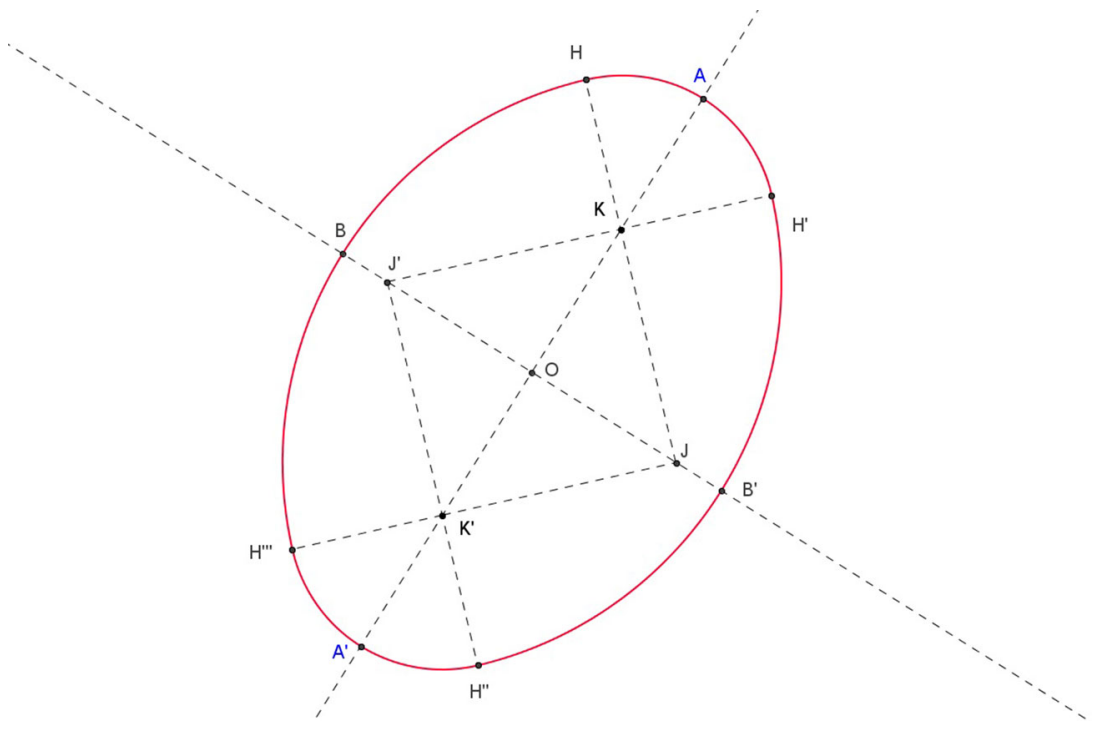

Fig. 21 A generic oval in a plane

Construction of Ovals 110 to 119 -given $B, B^{\prime}$ and any other two independent parameters other than $b$-follows the above guidelines for ovals 100 to 109 , with a similar numbering method. 


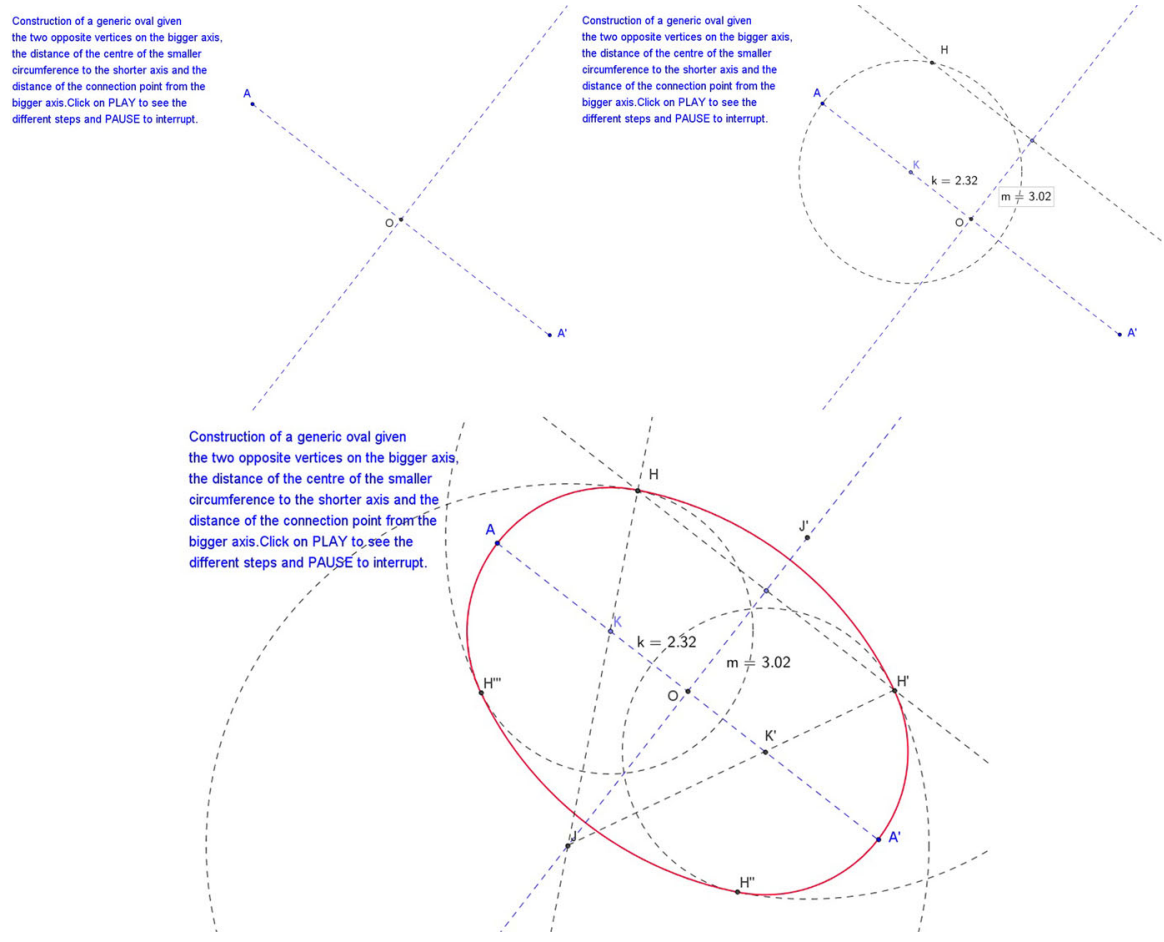

Fig. 22 Constructing Oval 106

When two non-opposite vertices are given the situation is more interesting, and we can make use of the properties of the CL listed and proved in "The Main Theorems on Ovals. Properties of the Connection Locus".

Given any two non-opposite vertices, say $A$ and $B$, a double CL is automatically determined, since Theorem 1 implies only two choices for $R$ on opposite sides of $A B$, leaving us only the choice of which side to choose for the centre $R$. We then have still two more parameters to choose, but we cannot use them both to choose $H$ because the connection point is linked to be on an arc of the CL. So we can still choose the symmetry centre $O$, which has to be on the half-circle with diameter $A B$ already containing point $R$. Here is how it is done.

Construction of Oval 120 (see Fig. 23) given any $A, B$ and then (feasible) $H$ and $O$ is the following (see link to Geogebra animation video on my website http:// www.mazzottiangelo.eu/en/pcc.asp):

- let $R$ and $R_{1}$ be the vertices of the two isosceles right triangles with hypotenuse $A B$, centres of a CL; choose one of them, say $R$, and choose any point $H$ on the $\operatorname{arc} A B$ with centre $R$

- let $P$ be the intersections of the parallel to $B H$ through $A$ with the circle having diameter $A B$, and $Q$ the intersection of the parallel to $A H$ through $B$ with the circle having diameter $A B$; choose $O$, the symmetry centre, on the arc $P Q$ 


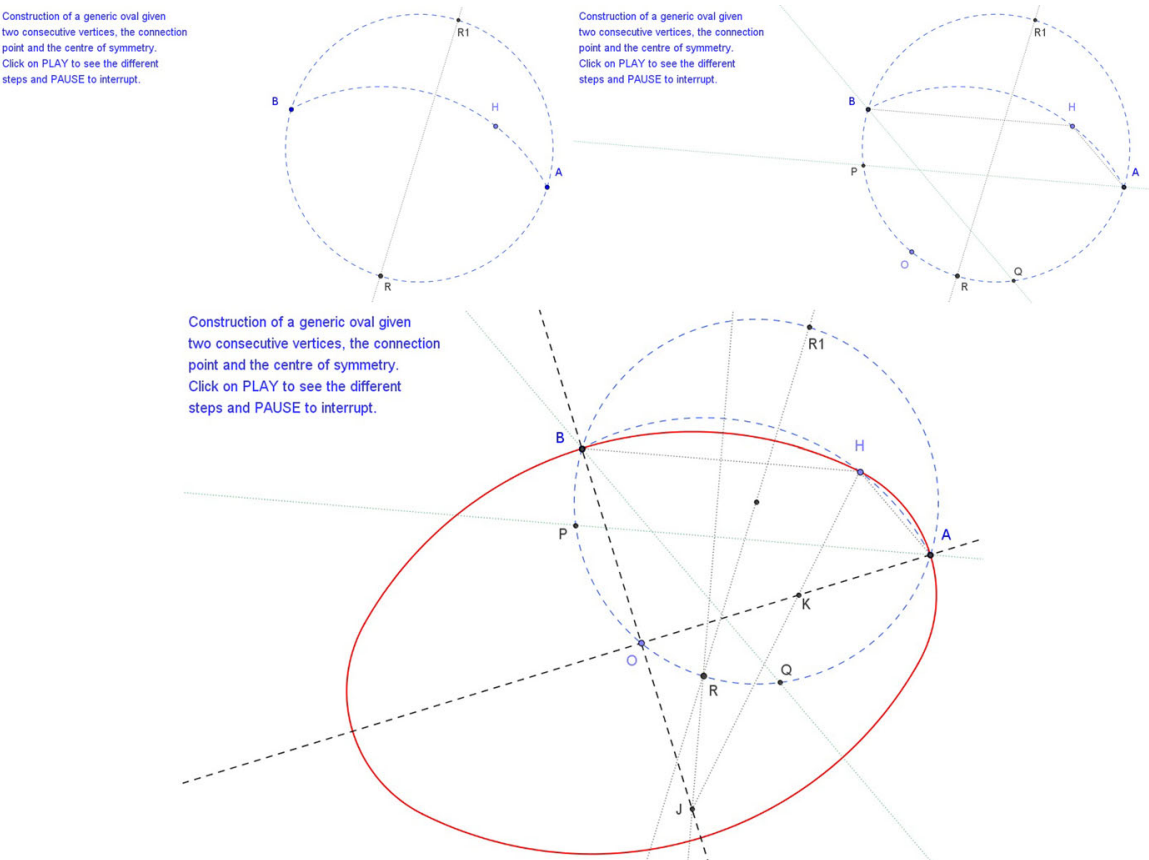

Fig. 23 Constructing Oval 120

- one way of drawing our arcs is now, for example, to find $J$ as the intersection of the axis of $B H$ with $O B$, and then $K$ as the intersection of $J H$ with $O A$

Limits for $O$ on the arc $P Q$ are based on construction evidence, not on mathematical proof. Choosing the centre of symmetry between $R$ and $Q$ instead of $R$ and $P$ exchanges the roles of $A$ and $B$.

If on the other hand one wants to choose $O$ first, then $H$ will have to be taken inside $A S$ (see Fig. 8).

It is also possible to choose either $K$ or $J$, after having chosen $A$ and $B$, each counting for two extra parameters, since they can freely be chosen inside two dimensional areas, as I have learned-although not proved-via construction evidence. See Ovals 121 and 122.

Construction of Oval 121 (see Fig. 24) given any $A, B$ and (feasible) $K$ is the following (see link to Geogebra animation video on my website http://www. mazzottiangelo.eu/en/pcc.asp):

- let $R$ and $R_{1}$ be the vertices of the two isosceles right triangles with hypotenuse $A B$; choose one of them, say $R$, draw the CL arc between $A$ and $B$, and then mirror the smaller of the two $R A$ arcs — of the circle with diameter $A B-$ w.r.t. the line $R A$

- choose any point $\mathrm{K}$ inside the just determined segment corresponding to the chord $R A$ 

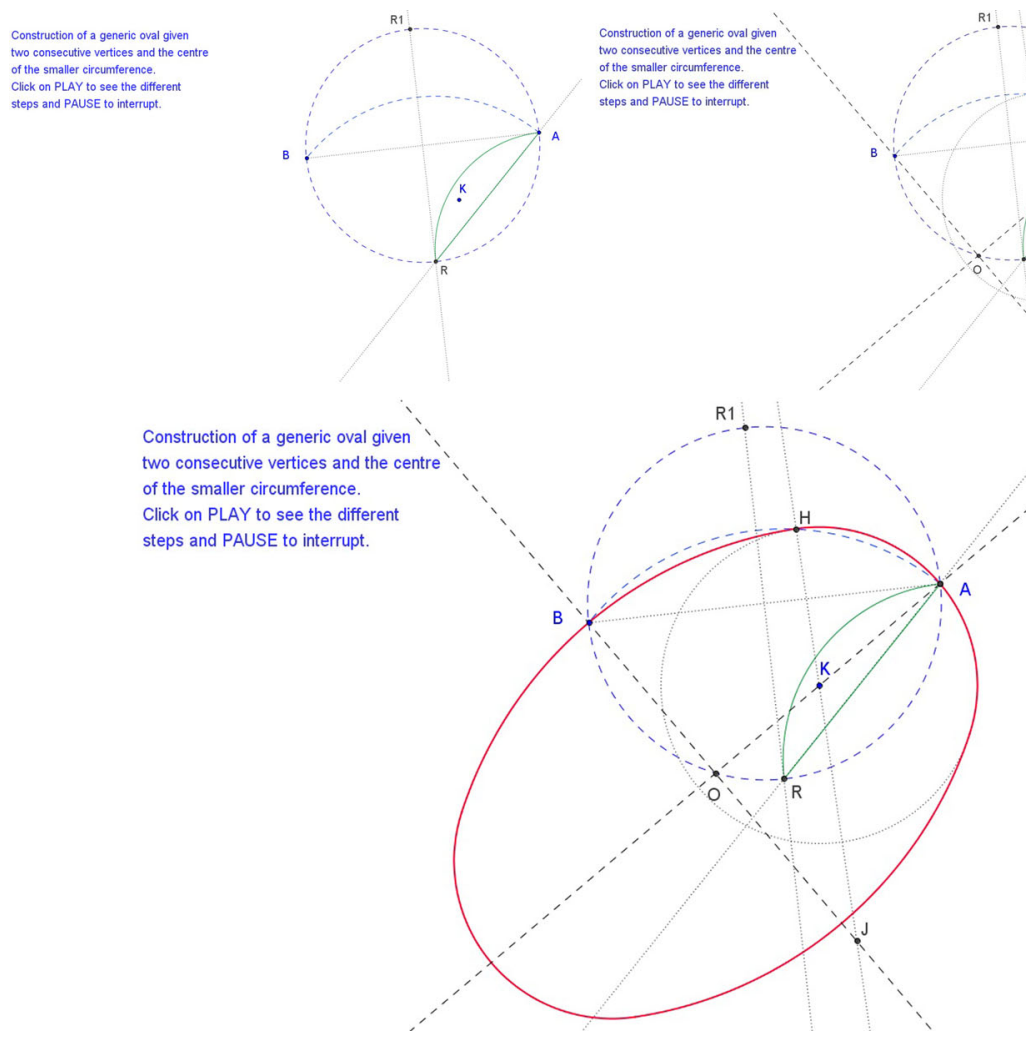

Construction of a generic oval given ' two consecutive vertices and the centre of the smaller circumference. Click on PLAY to see the different steps and PAUSE to interrupt.

Fig. 24 Constructing Oval 121

- $A K$ is now the symmetry axis, and the connecting point $H$ is the intersection between the CL with centre $R$ and the circle with centre $K$ and radius $A K$

- determine $O$ as the intersection between $A K$ and the circle with diameter $A B$, and then $J$ as the intersection of $O B$ - the other axis-with line $H K$ and so on.

Construction of Oval 122 (see Fig. 25) given any A, B and (feasible) $J$ is the following (see link to Geogebra animation video on my website http://www. mazzottiangelo.eu/en/pcc.asp):

- let $R$ and $R_{1}$ be the vertices of the two isosceles right triangles with hypotenuse $A B$; choose one of them, say $R$, draw the CL arc between $A$ and $B$, and then the line $R B$, forming an angle of $\frac{\pi}{4}$ with $R R_{1}$

- choose any point $\mathrm{J}$ inside the angle opposite to $B \hat{R} R_{1}$

- $J B$ is now the symmetry axis, $O$ is the intersection between $J B$ and the circle with diameter $A B$, and so on

Another choice for a starting pair is that of two centres of non-equal circles, say $J$ and $K$. Constructions are very straightforward and I will just outline the starting steps. Limitations for points are conjectured. 


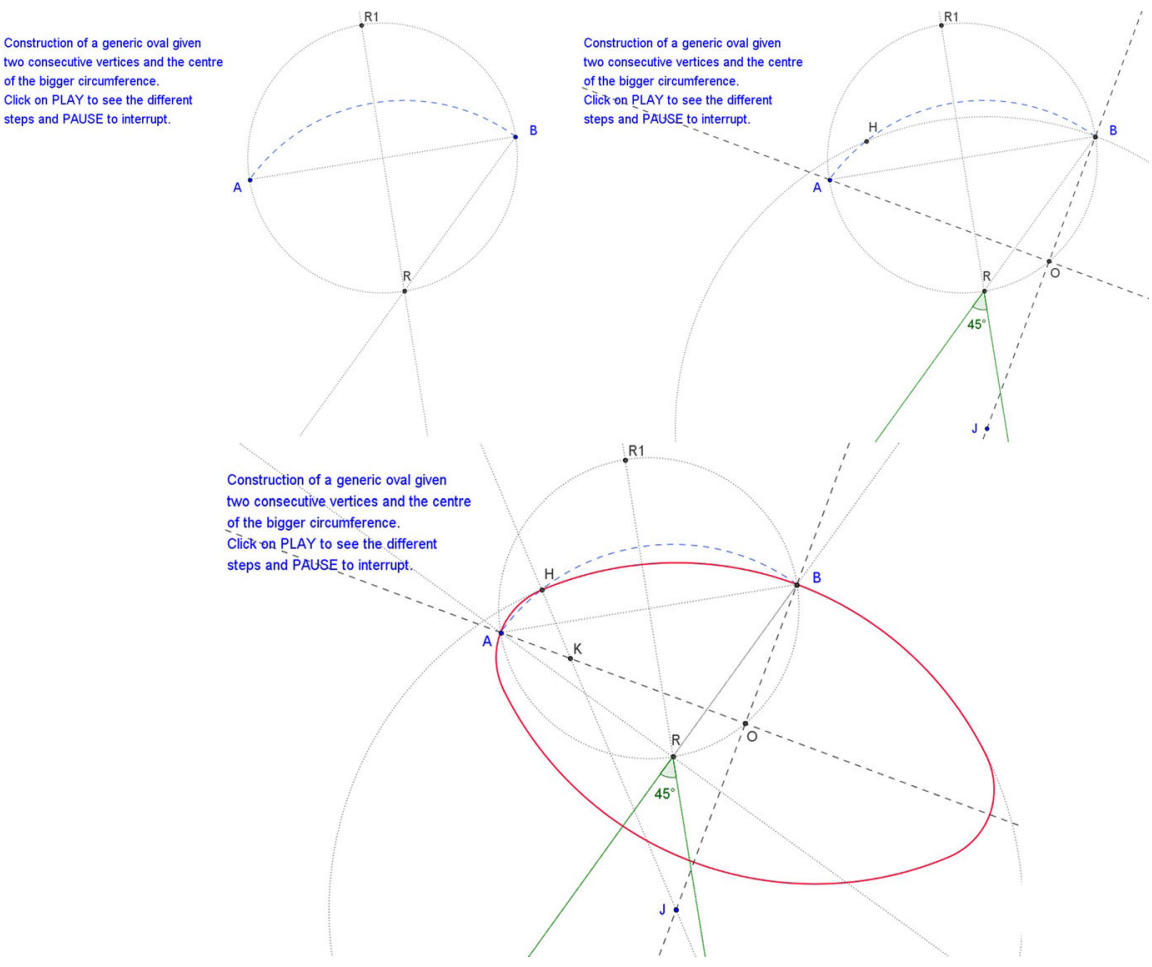

Fig. 25 Constructing Oval 122

Construction of Oval 130 (see Fig. 26a) given any $J$, K and $A$ begins as follows:

- choose any $J$ and $\mathrm{K}$

- then either

- $A$ inside the open half-plane determined by the perpendicular to $J K$ through $K$ not containing $J$, but not on line $J K$, or

- $A$ inside the open half-plane determined by the perpendicular to $J K$ through $K$ containing $J$, but not on $J K$, and outside of the circle with centre $J$ and radius $J K$

- draw the axis $K A$ and find $O$ as the intersection between $K A$ and the perpendicular to it from $J$; if $O$ lies between $A$ and $K$ what you have is actually $A^{\prime}$, and to get the "good" $A$ you need to mirror it w.r.t. $O$

Construction of Oval 131 (see Fig. 26b) given any $J, \mathrm{~K}$ and $B$ is equivalent to that of Oval 130:

- choose any $J$ and $\mathrm{K}$

- then choose $B$ inside the open half-plane determined by the perpendicular to $J K$ through $J$ containing $K$, excluding $J K$ and excluding the circle with centre $J$ and radius $J K$ 

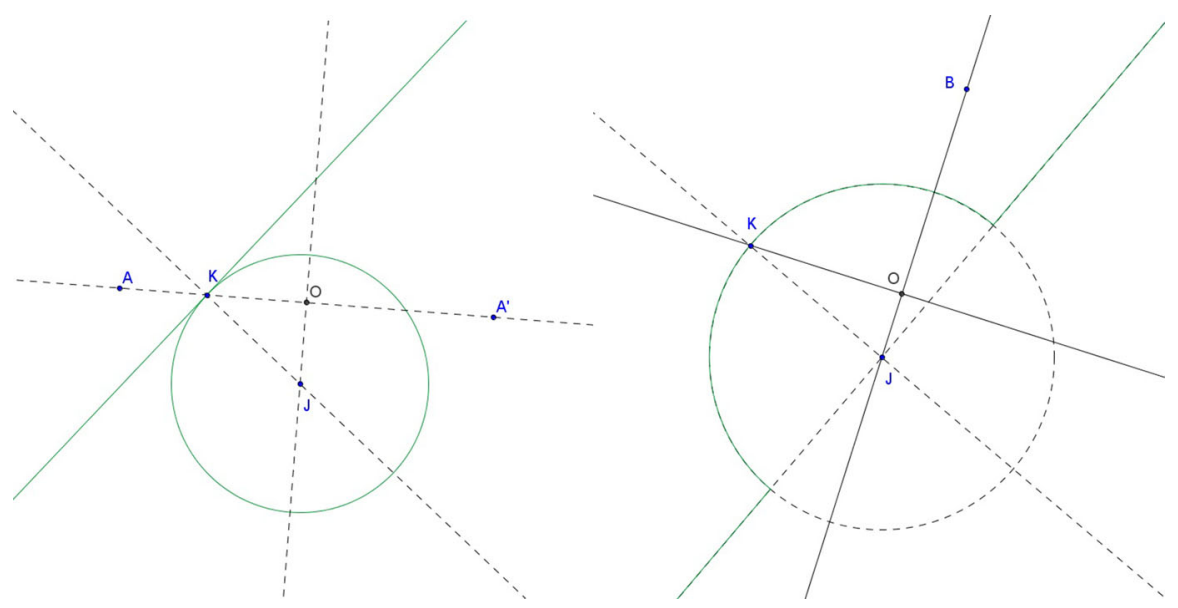

Fig. 26 a, b Initial steps for the constructions of Ovals 130 and 131

- draw the axis $B J$ and find $O$ as the intersection between $B J$ and the perpendicular to it from $K$; in half the cases you get a mirrored version of Fig. 21.

Construction of Ovals 132 to 134 (see Fig. 27) given any $J$, K, (feasible) $H$ and either $O$ or $A$ or $B$ begins as follows (see link to Geogebra animation video on my website http://www.mazzottiangelo.eu/en/pcc.asp):

- choose any $J$ and $\mathrm{K}$

- choose $H$ on $J K$ beyond $K$

- choose either

- $O$ on the circle with diameter $J K$ excluding $J$ and $K$, or

- $A$ on the open half circle $Y H Z$ excluding $H$, or

- $B$ on the open half circle $W H X$ excluding $H$

Constructions of Ovals 140 to 149 - given $K, K^{\prime}$ and any other two independent parameters other than $k$-follow the guidelines of "Ovals With Given Symmetry Axis Lines" once $O$ and the two axes have been drawn.

Constructions of Ovals 150 to 159-given $J, J^{\prime}$ and any other two independent parameters other than $j$-follow the guidelines of "Ovals With Given Symmetry Axis Lines" once $O$ and the two axes have been drawn.

As a last example I will consider a given tangent and tangent point, in addition to points $A$ and $K$. Bosse's construction is also used here.

Construction of Oval 160-given $K, A$, an extra point $C$ supposed to lie on the same half oval as $A$, and the tangent to the oval in $C$-is as follows (see Fig. 28; also see link to Geogebra animation video on my website http://www.mazzottiangelo.eu/en/pcc.asp):

- choose any $A$ and $\mathrm{K}$

- draw the circle with centre $K$ and radius $\overline{A K}$; let $D$ be the point opposite $A$

- draw the tangent through $D$ and the two other tangents perpendicular to the first one 
Fig. 27 Initial steps for constructing Ovals 132, 133 and 134

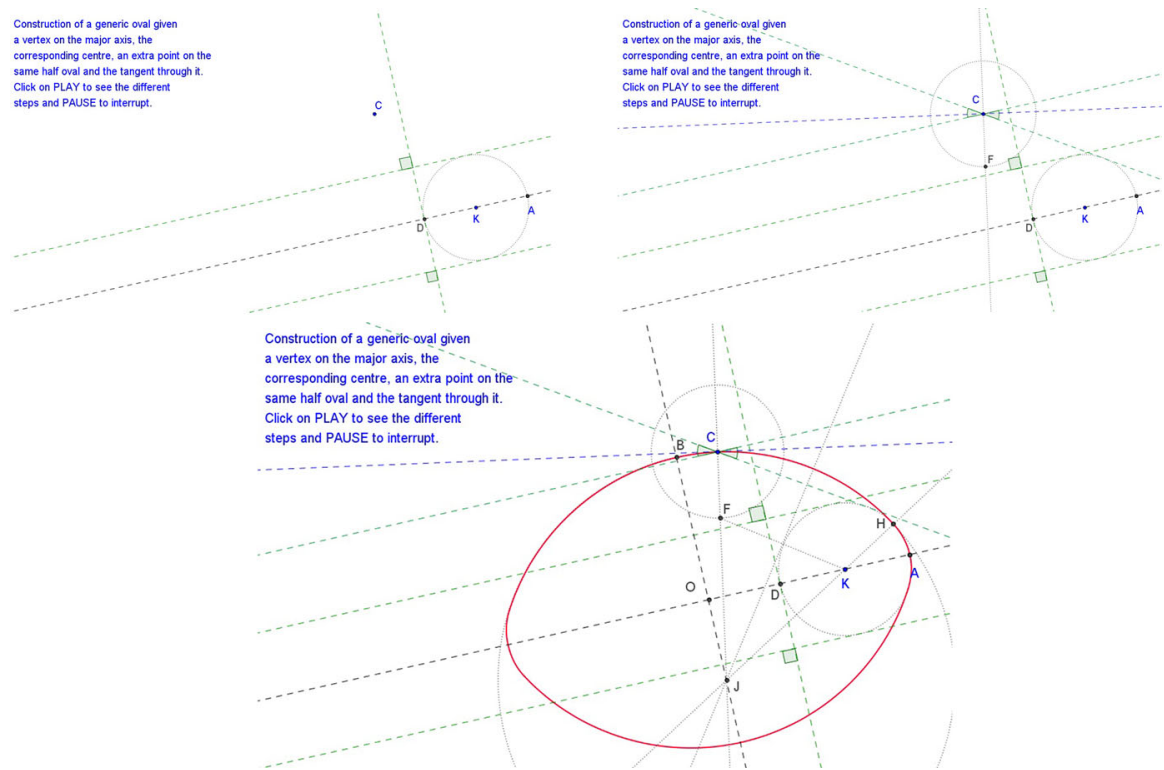

Fig. 28 Constructing Oval 160

- choose $C$ inside one of the right angles formed by the first and second, or the first and third tangent, in both cases opposite to the one containing the circle (see Fig. 28) 
- draw from $C$ the tangent to the first circle (the one on $A$ 's side), and the parallel to $K A$

- draw the desired tangent through $C$ between the two lines just drawn (see Fig. 28)

- take a segment $C F=K A$ on the normal line through $C$ (on the side of the first circle) find $J$ intersecting the normal line from $C$ and the axis of segment $K F$, etc.

Point $\mathrm{C}$ can be also chosen on the other side of the tangent through $\mathrm{D}$, but in that situation the possible choices for the tangent through $\mathrm{C}$ are more limited.

A lot more combinations of the parameters listed can be chosen, and constructions found, using the new tool of the CL and its properties along with the previously known properties and constructions.

\section{Conclusions and Further Developments}

I have shown in this paper that both the properties and the constructions of ovals presented here were at the time of Borromini very likely within reach, as were the lengthy but simple corresponding mathematical proofs behind them (see Mazzotti 2014). In addition to this, the tool of the CL has proved itself very useful for the purpose of drawing practically any kind of 4-centre oval.

Euclidean geometry has been used as a basis for this two-part work, the present paper and the one preceding it, at the beginning for historical reasons and then for consistency. But I have also found all the formulas yielding the different parameters of an oval in the twenty different cases listed in Fig. 9 by means of analytic geometry, as well as their limitations. These and other aspects of ovals, eggs and polycentric curves will be the subject of a future paper.

Acknowledgments I would like to thank Stefano Herzel for his friendship, help and encouragement, Felice Ragazzo for the long talks and the exchange of ideas and Sophie Püschmann for her constant support.

\section{References}

Bosse, A. 1655. Traité des Geometrales et Perspectives Enseignées dans l'Academie Royale de la Peinture et Sculpture. Paris: l'Auteur.

Canciani, M., Falcolini, C. and Spadafora, G. 2012. From complexity to geometrical rule. The case study of the dome of San Carlino alle Quattro Fontane in Rome. In Less More Architecture Design LandscapelLe vie dei Mercanti. Napoli: La Scuola di Pitagora ed.

Dotto, E. 2001. Note sulle costruzioni degli ovali a quattro centri. Vecchie e nuove costruzioni dell'ovale. Disegnare Idee Immagini XII 23: 7-14.

Dotto, E. 2002. Il Disegno Degli Ovali Armonici. Catania: Le Nove Muse.

Gentil Baldrich, J.-M. 1996. La traza oval y la sala capitular de la catedral de Sevilla. Una aproximación geométrica. in Quatro Edificios Sevillanos, eds. J.A. Ruiz de la Rosa et al., 77-147. Seville: Colegio Oficial de Arquitectos de Andalucía, Demarc. Occidental.

Hatch, J.G. 2002. The science behind Francesco Borromini's divine geometry. Nexus IV: Architecture and Mathematics: 127-139. Fucecchio, Florence: Kim Williams Books.

Huerta, S. 2007. Oval domes, geometry and mechanics. Nexus Network Journal 9(2):211-248. 
López Moto, A. 2011. Oval for any given proportion in architecture: a layout possibly known in the sixteenth century. Nexus Network Journal 13(3):569-597.

Mazzotti, A.A. 2014. A Euclidean approach to eggs and polycentric curves (in this issue). doi:10.1007/ s00004-014-0189-5.

Ragazzo, F. 1995. Geometria delle figure ovoidali. Disegnare idee immagini VI 11:17-24.

Ragazzo, F. 2011. Curve Policentriche. Sistemi di raccordo tra archi e rette. Roma: Prospettive.

Serlio, Sebastiano. 1619. Tutte l'Opere d'Architettura et Prospettiva di Sebastiano Serlio [...]. Venezia: G. de' Franceschi.

Simona, M. 2005. Ovals in Borromini's geometry. in Mathematics and Culture II Part 1, ed. M. Emmer, 45-52. Berlin: Springer.

Angelo Alessandro Mazzotti has a $\mathrm{PhD}$ in Operations Research (Dottore di Ricerca in Ricerca Operativa), and is a high school mathematics teacher and freelance mathematician. 